\title{
Complexity of functional connectivity networks in mild cognitive impairment subjects during a working memory task
}

\author{
Mehran Ahmadlou , Anahita Adeli , Ricardo Bajo , Hojjat Adeli
}

H I G H L I G H T S

- We use magneto-encephalograms in patients with mild cognitive impairment (MCI).

- We investigate complexity of functional connectivity network of MCI patients using two different measures: Graph Index Complexity and Efficiency Complexity.

- Efficiency Complexity is superior to Graph Index Complexity and its value at theta band can be used for diagnosis of MCI.

A B S T R A C T

Objectives: The objective is to study the changes of brain activity in patients with mild cognitive impairment (MCI). Using magneto-encephalogram (MEG) signals, the authors investigate differences of complexity of functional connectivity network between $\mathrm{MCI}$ and normal elderly subjects during a working memory task.

Methods: MEGs are obtained from 18 right handed patients with MCI and 19 age-matched elderly participants without cognitive impairment used as the control group. The brain networks' complexities are measured by Graph Index Complexity $\left(C_{r}\right)$ and Efficiency Complexity $\left(C_{e}\right)$.

Results: The results obtained by both measurements show complexity of functional networks involved in the working memory function in $\mathrm{MCl}$ subjects is reduced at alpha and theta bands compared with subjects with control subjects, and at the theta band this reduction is more pronounced in the whole brain and intra left hemisphere.

Conclusions: $C_{e}$ would be a better measurement for showing the global differences between normal and $\mathrm{MCl}$ brains compared with $C_{r}$

Significance: The high accuracy of the classification shows $C_{e}$ at theta band can be used as an index for assessing deficits associated with working memory, a good biomarker for diagnosis of $\mathrm{MCl}$.

\section{Introduction}

Mild Cognitive Impairment (MCI) is a heterogeneous cognitive disorder defined as mild deficits in cognitive functions including episodic memory impairment, greater than expected by age (Petersen et al., 2001; Adeli et al., 2005a,b, 2008). Prevalence of 
$\mathrm{MCl}$ in elderly population ( $50-95$ years) is between $5.8 \%$ and $18.5 \%$ (Collie and Maruff, 2000). MCI patients have a high conversion rate, approximately $50 \%$, to dementia, and hence $\mathrm{MCl}$, as a transitional state between healthy aging and dementia, is thought as an important target of treatment for dementia (Choi et al., 2009; Villeneuve et al., 2009; Ally et al., 2009).

Working memory refers to the structures and processes involved in temporarily holding information in mind and manipulating them for further processing (Baddeley and Hitch, 1974; Patalong-Ogiewa et al., 2009; Ramanathan et al., 2012). Impairment of working memory results in impaired executive function in $\mathrm{MCl}$. As such, investigation of working memory deficits in $\mathrm{MCI}$ is of vital importance for understanding of the pathology of $\mathrm{MCI}$ and predicting dementia (Collie and Maruff, 2000; Wenger et al., 2010). MRI-based studies have found gray matter loss in medial temporal, parietal and frontal regions in amnestic $\mathrm{MCI}$ patients (defined as $\mathrm{MCI}$ patients with impaired episodic and working memory) (Karas et al., 2004; Barbeau et al., 2008). In a PET study on word-pair learning Moulin et al. (2007) suggested different activation patterns in $\mathrm{MCl}$ patients compared with normal elderly subjects. During incremental learning the MCI subjects showed increased activity in the occipital regions (instead of change in frontal activations, which occurs in normal subjects) and during retrieval they showed increased activity in the left frontal region. Patalong-Ogiewa et al., 2009) found MCI patients have impairments not only in episodic memory but also in working memory which shows the deficit is not restricted only to hippocampus but is more global.

In summary, the literature shows the changes of brain activity of $\mathrm{MCI}$ patients, observed in a few neuroimaging studies, have been quite variable (Gronholm et al., 2007). Hence studying the impairment of brain networks during working memory tasks is of great interest. Such a study may result in discovery of unique markers for all or specific subsets of $\mathrm{MCI}$ patients to be used to identify those who will develop dementia. In a recent article Ahmadlou and Adeli (2011a) presented a new methodology for investigation of the structure of the brain functional connectivity and understanding of the brain based on graph theory and community pattern analysis of functional connectivity graph of the brain obtained from encephalograms (EEGs). The methodology consists of three main parts: fuzzy synchronization likelihood (FSL), community partitioning, and decisions based on partitions. Using magneto-encephalogram (MEG) signals, in this research the authors investigate differences of complexity of functional connectivity network, a global property of the brain, between $\mathrm{MCI}$ and normal elderly subjects during a working memory task. Complexity of functional connectivity network is used to indicate how the neuronal regions interact with each other (how much dense/sparse [in the sense of ratio of the connectivity to the number of brain regions], and random/ordered [in the sense of distribution of the connectivity among the brain regions: how much the regions are randomly connected and how much the connectivity is equal between each brain region and the others]) and to determine the efficiency of the network in transmitting information. The higher the complexity during the working memory task, the higher the efficiency of the brain network in transmitting the information associated with the working memory.

In this research, the complexity is computed using two different measures: Graph Index Complexity and Efficiency Complexity (Kim and Wilhelm, 2008; Bello-Orgaz et al., 2012). These measures have been selected because they can measure the global complexity of the brain functional connectivity network after quantifying the constructed network using synchronization methods (Besio et al., 2011; Han et al., 2011; Serletis et al., 2013; Rangaprakash et al., 2013). The global complexity quantifies the structure of connections in a network and determines where it is between random and rigid structures. Complexity in the brain network is a vital characteristic for feasible synchronization and efficient information transmission (Ahmadlou et al., 2012a, 2013). This means having a short and strong pathway between brain regions so that the information can flow easily with optimum number of connections. The common concept of correlation or connectivity between brain regions is just a local concept. If all brain regions connect to each other with equally strong connections, the global network may not be efficient for transmission of the information from one region to another region (since it is paying a lot of extra cost/connections). Indeed some areas are hubs (usually high level processing regions) and need more connectivity and other areas do not need high connectivity. It is the distribution of these connectivities among the brain regions that determines the efficiency of the brain network.

The Graph Index Complexity has been used in other branches of sciences usually to study complexity of social and economic networks. The authors were the first researchers to introduce the concept of visibility graph in neuroscience, but for measuring the complexity of the EEG signals and not for measuring the global complexity of functional brain networks (Ahmadlou et al., 2010). They utilized the Graph Index Complexity to measure complexity of the EEG signals. The Efficiency Complexity has been used in the neuroscience literature as one of the Small-World Network components (Watts and Strogatz, 1998; Han et al., 2011), but in this research it is used as an index of complexity of network irrespective of the concept of Small-World Network.

Many studies show oscillations as well as deficits in theta and alpha frequency bands during working memory tasks in healthy subjects and also MCI patients (Klimesch, 1999; Stam, 2000; Bastiaansen et al., 2002; Schmiedt et al., 2005; Deiber et al., 2009; Khader et al., 2010; Morgan et al., 2013). Hence in this study the complexity of functional connectivity networks during the working memory task is analyzed in the theta and alpha frequency bands.

\section{Methods}

\subsection{Participants}

All subjects or legal representatives provided written consent to participate in the study, which was approved by the local ethics committee of the Hospital Clínico Universitario San Carlos. Eighteen right handed patients with $\mathrm{MCI}$ were recruited from the Geriatric Unit of the "Hospital Clínico Universitario San Carlos Madrid". In addition, nineteen age-matched elderly participants without cognitive impairment were included as the control group. Age and years of education were matched to the MCI group (for details see Bajo et al., 2010,2012; the same subjects were used in that study): 10 years for the MCI group and 11 years for controls. To confirm the absence of memory complaints, a score of 0 was required in a 4-question questionnaire (see Mitchell, 2008). None of the participants had a history of neurological or psychiatric condition.

The diagnosis of $\mathrm{MCI}$ was made according to previously published criteria proposed by Petersen et al. (Grundman et al., 2004; Petersen, 2004). Thus, MCI patients fulfilled the following criteria: (1) cognitive complaint corroborated by an informant (a person who stays with the patient at least for half a day 4 days a week); (2) objective cognitive impairment documented by delayed recall in the Logical Memory II subtest of the Wechsler Memory Scale-III-Revised (score $\leqslant 16 / 50$ for patients with more than 15 years of education; score $\leqslant 8 / 50$ for patients with $8-15$ years of education); ( 3 ) normal general cognitive function as assessed by a clinician during a structured interview with the patient and an informant and, additionally, a Mini Mental State Examination (MMSE) score greater than 24 ; (4) relatively preserved daily living 
activities as measured by the Lawton scale; (5) not sufficiently impaired, cognitively or functionally, to meet criteria for dementia. Age and years of education were matched to the $\mathrm{MCI}$ group. According to their clinical and neuropsychological profile, all subjects in this group met criteria for multi-domain $\mathrm{MCl}$ (Petersen, 2004). None of the subjects with MCI had evidence of depression as measured using the geriatric depression scale (score lower than 9) (Yesavage and Brooks, 1991).

$\mathrm{MCl}$ subjects and healthy participants underwent a neuropsychological assessment in order to establish their cognitive status with respect to multiple cognitive functions. Specifically, memory impairment was assessed using the Logical Memory immediate (LM1) and delayed (LM2) subtests of the Wechsler Memory Scale-III-Revised. Two scales of cognitive and functional status were applied as well: the Spanish version of the MMSE (Lobo et al., 1979), and the Global Deterioration Scale/Functional Assessment Staging (GDS/FAST). It should be noted that the data used in this research are sufficient to make general diagnosis of $\mathrm{MCl}$. For the purposes of this paper the MCI subtype is not critical.

\subsection{Sternberg task}

Sternberg Task (Sternberg, 1966) is a well-known task for studying working memory, used especially in MEG studies (Tuladhar et al., 2007; Deeny et al., 2008; Maestú et al., 2008; Stephane et al., 2010; Brookes et al., 2011; Kurimoto et al., 2012). Magnetoencephalography (MEG) scans were obtained in the context of a modified version of the Sternberg's letter-probe task (deToledoMorrell et al., 1991; Maestú et al., 2001) in which a set of five letters was presented and subjects were asked to keep the letters in mind. After the presentation of the five-letter set, a series of single letters ( $1000 \mathrm{~ms}$ in duration with a random ISI between 2 and $3 \mathrm{~s}$ ) was introduced one at a time, and participants were asked to press a button with their right hand when a member of the previous set was detected (Fig. 1). The list consisted of 250 letters in which half were targets (previously presented letters), and half distracters (not previously presented letters). Subjects undertook a training series before the actual test. The subject's responses were classified into four different categories according to the subject's performance in the experiment: hits, false alarms, correct rejections and omissions. Only hits were considered for further analysis because the authors were interested in evaluating the functional connectivity patterns which support recognition success. The

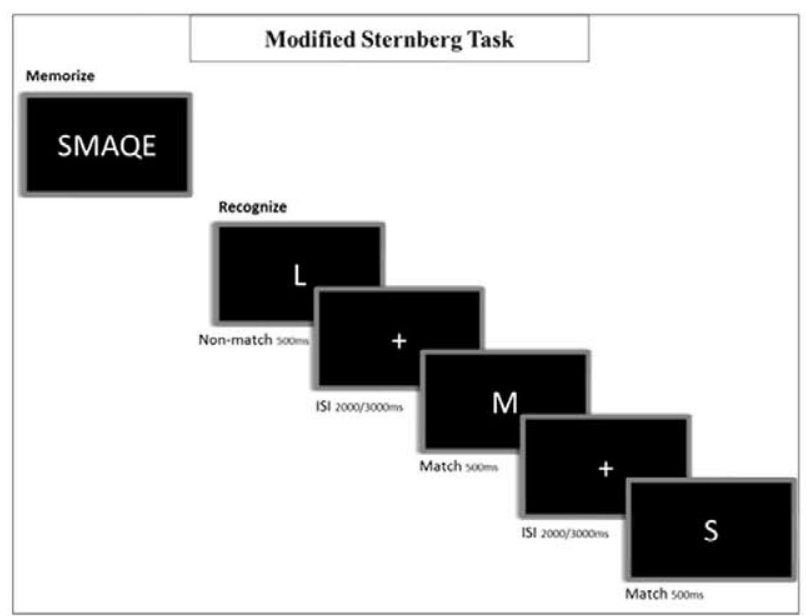

Fig. 1. Representation of the memory task paradigm. In the encoding phase, participants are instructed to memorize 5 letters, that is "SMAQE". In the recognition phase, participants are instructed to make a match/non-match button-press to indicate that the presented letter matched any of the encoded ones.

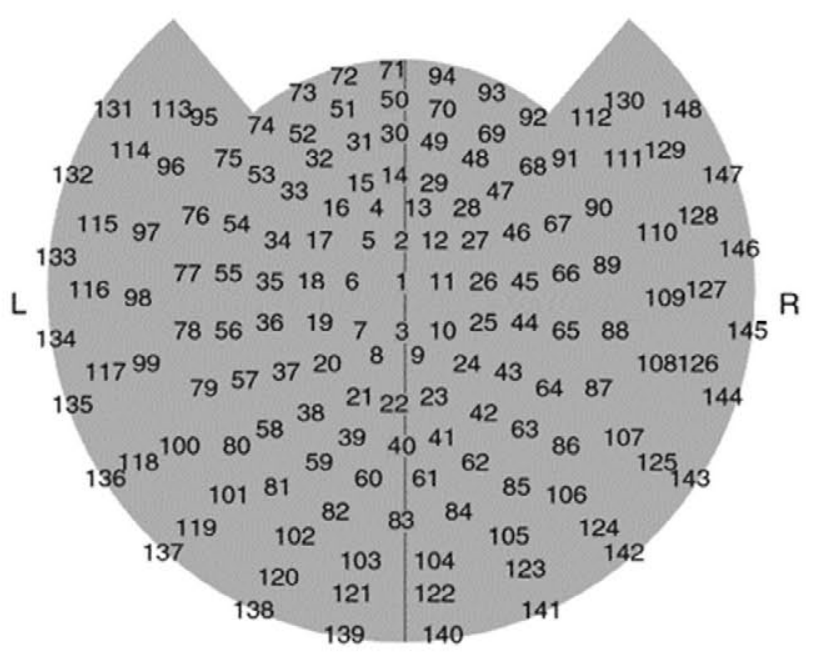

Fig. 2. The brain map of the 148 loci where MEG is recorded. The vertical line partitions the whole brain into the left $(\mathrm{L})$ and right $(\mathrm{R})$ hemispheres.

percentage of hits ( $80 \%$ control group and $84 \% \mathrm{MCI}$ group) and correct rejections (92\% control group and $89 \% \mathrm{MCI}$ group) was high enough in both groups, indicating that participants actively engaged in the task. No significant difference between the two groups during the memory task was revealed between groups, either with respect to the targets (total number of hits, misses and reaction time), or to the distracters (correct rejections, false alarms and reaction time).

\section{3. $M E G$ recording}

The 148-channel MEG (Fig. 2) signals were recorded with a $254 \mathrm{~Hz}$ sampling frequency and a band pass of $0.5-50 \mathrm{~Hz}$, using a 148-channel whole-head magnetometer (MAGNES $2500 \mathrm{WH}, 4-\mathrm{D}$ Neuroimaging) confined in a magnetically shielded room in order to reduce the environmental noise. All MEG recordings were conducted in the morning between 9:00 A.M. and 12:00 A.M. During the MEG register subjects performed the Sternberg memory task. Fig. 3 shows samples of MEG signals at three locations in the frontal, central, and parietal lobes for an MCI (left: a, b, and c, respectively) and a normal (right: d, e, and f, respectively) subject. An environmental noise reduction algorithm using reference channels at a distance from the MEG sensors was applied to the data. Thereafter, single trial epochs were visually inspected by an experienced investigator, and epochs containing visible blinks, eye movements or muscular artifacts were excluded from further analysis. All time epochs had one second length without baseline (baseline was not studied). Since the MEG signals have a complex dynamic, neurologists are not able to read MEGs or find visual markers. Our research provides a methodology to reveal the differences between the two groups.

All data were converted to MATLAB format (.mat). The MATLAB data of each subject contains a $3-D$ matrix $\{148$ (number of channels) $\times 230$ (number of sample per epoch) $\times$ number of epochs\}; the number of epochs per subject varies between 29 and 100 epochs (some records are discarded because of artifacts).

\subsection{Functional connectivity}

Measurements of synchronizations between different brain signals are quantified interdependencies between the associated neuronal regions. Synchronization measurements are classified in two general categories: linear methods and nonlinear methods. The authors have recently used nonlinear synchronization techniques 
a

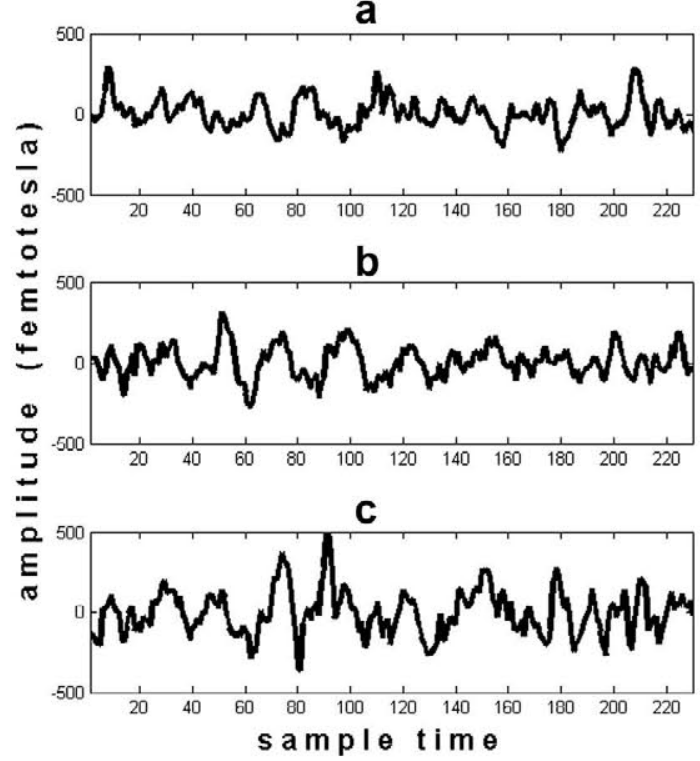

d

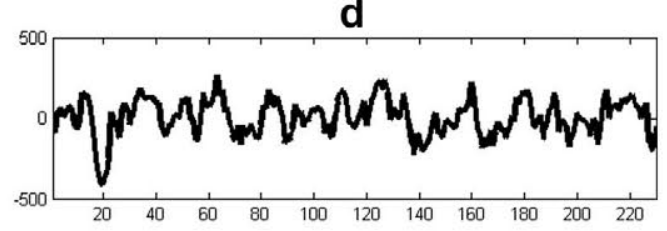

e

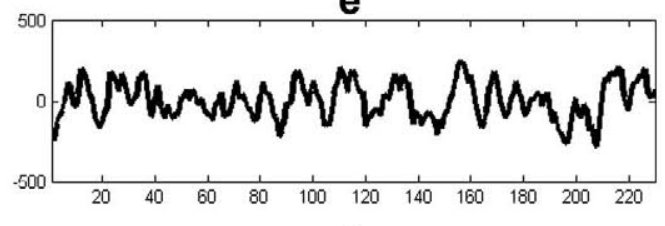

f

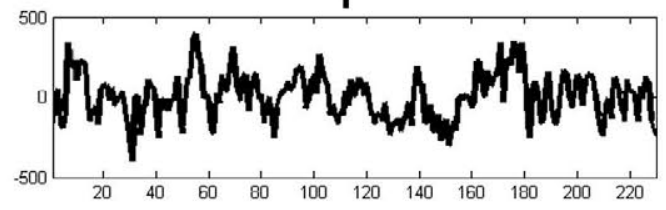

Fig. 3. Samples of MEG signals at three locations in frontal, central, and parietal lobes for an MCl (left: a-c, respectively) and a normal (right: d-f, respectively) subject.

such as synchronization likelihood (introduced by Stam and van Dijk (2002), fuzzy synchronization likelihood (introduced by Ahmadlou and Adeli (2011b), and visibility graph similarity (introduced by Ahmadlou and Adeli (2012)) for diagnosis of attention deficit/hyperactivity disorder (ADHD) (Ahmadlou and Adeli, 2010b, 2012; Ahmadlou and Adeli, 2011a,b; Ahmadlou et al., 2012a), Autism Spectrum Disorder (ASD) (Ahmadlou et al., 2012b), and Major Depressive Disorder (MDD) (Ahmadlou et al., 2012c). A large number of sequential sample times is needed for nonlinear synchronization measurements (usually more than 10,000 sample times). Each epoch of the MEG signal to be analyzed contains only 230 sample times which precludes the application of nonlinear synchronization methods. As such, in this research the functional connectivity analysis is accomplished through a linear method, the classic cross-correlation function.

\subsection{Functional connectivity network}

A functional connectivity matrix is constructed using the correlation coefficients between signals of all pair-wise combinations of the channels. Fig. 4 shows how an example 148-channel MEG set is transformed to its functional connectivity network. Computed correlation coefficients between channel pairs are entries of a $148 \times 148$ connectivity matrix. Indeed the connectivity matrix is the adjacency matrix of the weighted connectivity network. Fig. $4 \mathrm{a}$ and $\mathrm{b}$ show the grand average connectivity matrices of normal and $\mathrm{MCl}$ subjects, respectively. The light intensity of each array indicates the strength of the connectivity. The darker intensity indicates a lower value in the connectivity matrices. Fig. 4a and b illustrate the average connectivity matrices in the networks of the whole brain, left hemisphere, and right hemisphere of the healthy and $\mathrm{MCI}$ groups. In the next step the complexity of these networks will be computed for comparison.

\subsection{Measuring complexity of networks}

In the past decade or so, two measures have been proposed in the statistics literature for measuring complexity of a given unweighted graph (a graph whose edges have only two value, 0 and 1) with $N$ nodes (Kim and Wilhelm, 2008). They are described in Section 2.6. The original functional connectivity graphs obtained by neuroimaging tools are weighted graphs, but researchers often simplify them by converting them to a binary or unweighted graph using a threshold. In doing so significant information is lost. Since weights of connectivity graph contain a major part of its structural information (Barrat et al., 2004; Li et al., 2007) the authors avoid converting it to a binary graph. In this study two complexity measures are used for a given weighted graph (whose edges have real values in the range of 0 and 1) with $N$ nodes.

\subsubsection{Graph Index Complexity $C_{r}$}

Let $r$ be the largest eigenvalue of the adjacency matrix of a weighted or unweighted graph. The Graph Index Complexity, $C_{r}$, indicates the diversity or heterogeneity of distribution of the edges over all the nodes, defined as (Kim and Wilhelm, 2008):

$C_{r}=4 c(1-c)$

where:

$c=\frac{r-2 \cos (\pi /(N+1))}{N-1-2 \cos (\pi /(N+1))}$

The index $C_{r}$ varies between 0 and 1: the more complex the graph's structure, the larger will be $C_{r}$.

\subsubsection{Efficiency Complexity $C_{e}$}

Efficiency of a weighted graph is computed by the short path length (the shortest path connecting the pairs edges) of the graph (the shorter the path length, the higher the efficiency) which indicates the speed of information transition from a node to another node of the graph. It has been shown that many social and natural networks are of the so-called small-world networks, which means coexistence of small short path lengths between nodes with relatively sufficient number (and not too many more than the sufficient number) of connections between the nodes. There is a positive correlation between the efficiency of a network and the number of its edges. The efficiency of a graph is defined as (Latora and Marchiori, 2003) (They used it as a characteristic of smallworld network, but we are using it as a characteristic of complexity of networks):

$$
C_{e}=\frac{1}{N(N-1) / 2} \sum_{1 \leqslant j<i \leqslant N}\left(\frac{1}{d_{i j}}\right)
$$




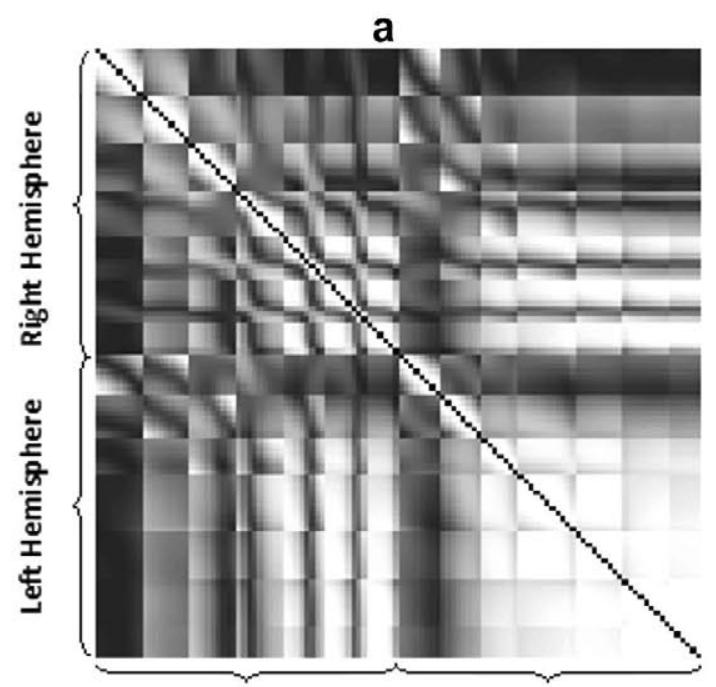

Right Hemisphere Left Hemisphere

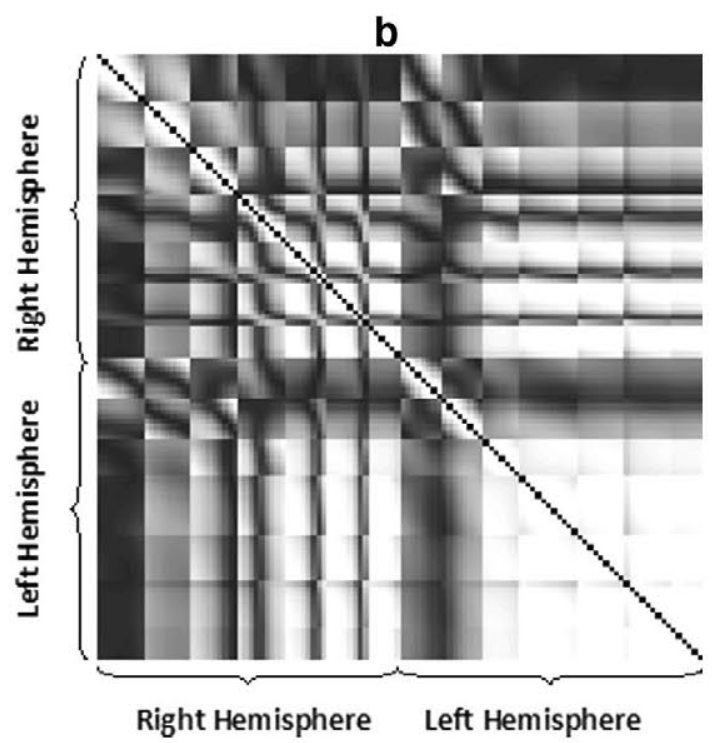

Fig. 4. Grand average connectivity matrix of the (a) normal subjects and (b) MCI subjects.

where $d_{i j}$ is defined as inverse of the weight between $i$ th node and $j$ th node. The index $C_{e}$ is presented as an overall measure of routing efficiency characteristics of the graph and information transmission flexibility of the network. In this research, $C_{e}$ is a measure of the extent of average connectivity or overall routing efficiency and flexibility of the neocortex network which helps different neuronal areas of the network to be synchronized with each other in different situations. This feasibility in synchronization is a key point in information transmission and accomplishment of all behavioral and cognitive activities (Ahmadlou et al., 2012a). In this way efficiency of functional connectivity matrices, which correspond to neuronal network's structures in the alpha $(8-12 \mathrm{~Hz})$ and theta (4-8 Hz) sub-bands, are computed.

\subsection{Statistical analysis and classification}

The one-way Analysis of Variance (ANOVA) was used to evaluate the ability of the $C_{e}$ and $C_{r}$ indices at theta and alpha bands to discriminate between normal and $\mathrm{MCI}$ groups. Next, the Enhanced Probabilistic Neural Network (EPNN) developed by the authors recently (Ahmadlou and Adeli, 2010a) as an improved version of PNN (Adeli and Panakkat, 2009; Lopez-Rubio et al., 2011) and a power- ful classification technique is used to classify the two groups based on the most discriminating features. Local decision circles enable EPNN to incorporate local information and non-homogeneity existing in the training population. The circle has a radius which limits the contribution of the local decision. Two parameters, the spread parameter and the radius of local decision circles, are optimized to maximize the performance of the model. Inputs of the EPNN are the features discovered by the one-way ANOVA in the previous step. EPNN consists of an input layer with number of nodes equal to the number of the selected features, a pattern layer which computes the likelihood of the input data to each training data using two local and global Bayesian rules, a summation layer which computes the likelihood of the input data to each class (in this work, $\mathrm{MCI}$ and healthy), and an output layer with one node which assigns the input data to the class with maximum likelihood. The number of nodes in the pattern layer is equal to the number of training data sets and the number of nodes in the summation layer is the same as the number of classes (i.e. 2). The EPNN has shown successful results in previous studies for diagnosis of autism, cerebral palsy, and major depression (Ahmadlou et al., 2012c,d; Sajedi et al., 2013).

\section{Results}

\subsection{Clinical evaluations}

Table 1 summarizes the demographic and clinical information (age, MMSE, GDS, LM1, and LM2) for both groups. The data set used in this study did not include any information about premorbid IQ smoking, ApoE typing and the head CT or MRI images from the subjects ( $\mathrm{MCI}$ and Control). The authors acknowledge that this information would have made the study more valuable.

The MCI group performed significantly worse on tests of memory, i.e., LM1 and LM2, than normal controls $(p<0.0001)$. The fact that the sample of MCI subjects' data used in this research came from a memory clinic rather than from a population-based sample explains the high proportion of amnestic MCI.

\subsection{Graph Index Complexity $C_{r}$}

Table 2 shows the mean and standard deviation of $C_{r}$ for the whole brain, intra left and right hemispheric, and inter hemispheric neuronal networks (see Fig. 2) at alpha and theta bands, as well as the associated ANOVA $p$-values and $F$-values in discrimination of the $\mathrm{MCI}$ and healthy elderly groups. It shows $C_{r}$ of the whole brain at alpha band is significantly different between the two groups ( $p$-value $<0.05$ ), while the ability of $C_{r}$ in the whole brain and intra left hemisphere in distinguishing the two groups at theta band is much higher ( $p$-value $<0.0001$ ). Also at the theta band the $C_{r}$ of the inter-hemispheric network is significantly decreased in $\mathrm{MCI}$ group ( $p$-value $<0.05$ ). Fig. 5 shows distribution of $C_{r}$ of the brain neuronal network at (a) alpha and (b) theta; (c) $C_{r}$

Table 1

Demographic and clinical information of the control and MCI groups (Bajo et al. 2010).

\begin{tabular}{|c|c|c|c|c|c|c|}
\hline & Sample (sex) & Age & MMSE & GDS & LM1 & LM2 \\
\hline Control & $\begin{array}{l}19 \text { (12 } \\
\text { Female) }\end{array}$ & $71.6 \pm 8$ & $29.5 \pm 0.7$ & 1 & $42.5 \pm 8^{*}$ & $26.7 \pm 7$ \\
\hline $\mathrm{MCI}$ & $\begin{array}{l}18 \text { (11 } \\
\text { Female) }\end{array}$ & $74.8 \pm 3$ & $27.7 \pm 1$ & 3 & $19.1 \pm 5$ & $13.1 \pm 6$ \\
\hline
\end{tabular}

MMSE: Mini Mental State Exam (maximum score is 30); GDS: Global Deterioration Scale; LM1: Logical Memory immediate recall; LM2: Logical Memory delayed recall. * $p$-value for distinguishing the control and $\mathrm{MCl}$ groups is significant at the 0.0001 level. 
Table 2

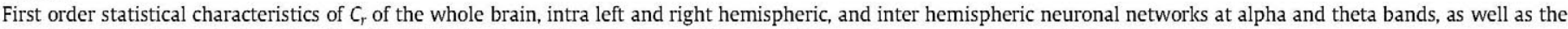
associated ANOVA p-values and F-values in discrimination of the two groups (M. and Std. stand for mean and standard deviation, respectively).

\begin{tabular}{|c|c|c|c|c|c|c|c|c|c|}
\hline \multirow[t]{2}{*}{ Band } & \multirow[t]{2}{*}{ Subjects } & \multicolumn{2}{|l|}{ Whole brain } & \multicolumn{2}{|c|}{ Intra left hemispheric } & \multicolumn{2}{|c|}{ Intra right hemispheric } & \multicolumn{2}{|c|}{ Inter hemispheric } \\
\hline & & M. \pm Std. & $p$-val. $(F)$ & M. \pm Std. & $p$-val. $(F)$ & M. \pm Std. & p-val. $(F)$ & M. \pm Std. & $p$-val. $(F)$ \\
\hline Alpha & $\begin{array}{l}\text { Normal } \\
\mathrm{MCl}\end{array}$ & $\begin{array}{l}.9828 \pm .009 \\
.9866 \pm .007\end{array}$ & $.0246^{*}(5.51)$ & $\begin{array}{l}.9779 \pm .012 \\
.9814 \pm .008\end{array}$ & $.1066(2.75)$ & $\begin{array}{l}.9908 \pm .009 \\
.9928 \pm .005\end{array}$ & $.1023(2.82)$ & $\begin{array}{l}.9875 \pm .010 \\
.9900 \pm .006\end{array}$ & $.0872(3.11)$ \\
\hline Theta & $\begin{array}{l}\text { Normal } \\
\mathrm{MCI}\end{array}$ & $\begin{array}{l}.867 \pm .050 \\
.832 \pm .039\end{array}$ & $5.8134 \times 10^{-5^{* *}}(21.12)$ & $\begin{array}{l}.710 \pm .041 \\
.693 \pm .029\end{array}$ & $9.6811 \times 10^{-5^{* *}}(19.50)$ & $\begin{array}{l}1.023 \pm .053 \\
.971 \pm .037\end{array}$ & $.5329(.41)$ & $\begin{array}{l}.994 \pm .057 \\
.933 \pm .052\end{array}$ & $.0119^{*}(7.06)$ \\
\hline
\end{tabular}

- Correlation is significant at the 0.05 level.

** Correlation is significant at the 0.01 level.

a

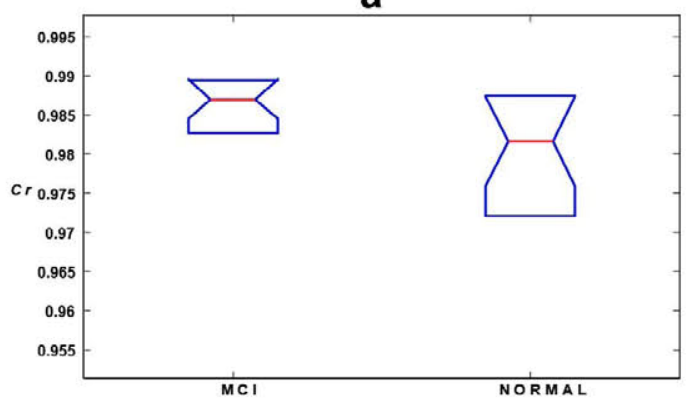

C

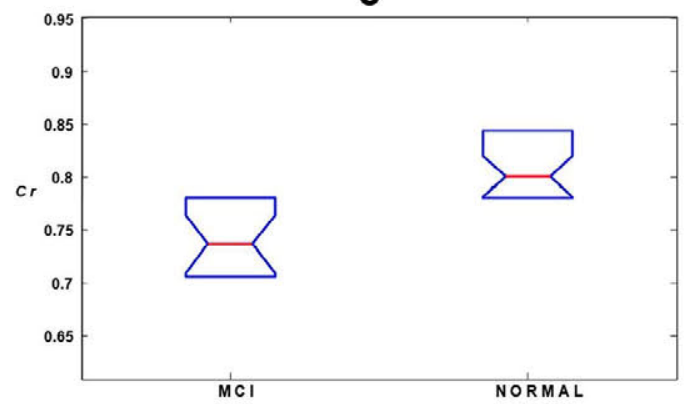

b

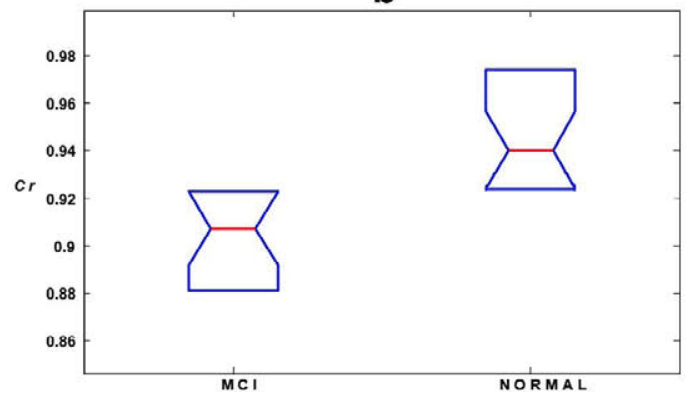

d

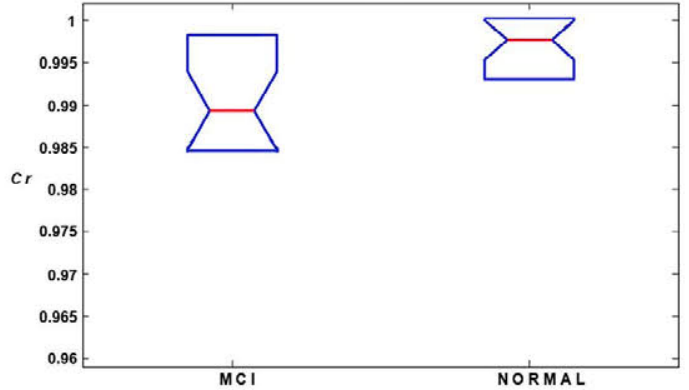

Fig. 5. $C_{r}$ of brain neuronal network at (a) alpha and (b) theta; (c) $C_{r}$ of intra left hemispheric network at theta and (d) $C_{r}$ of inter hemispheric network at theta.

Table 3

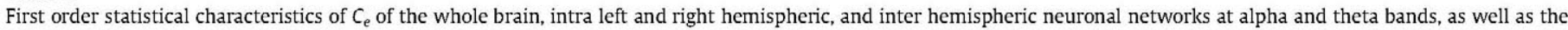
associated ANOVA p-values and $F$ values in discrimination of the two groups (M. and Std. stand for mean and standard deviation, respectively).

\begin{tabular}{|c|c|c|c|c|c|c|c|c|c|}
\hline \multirow[t]{2}{*}{ Band } & \multirow[t]{2}{*}{ Subjects } & \multicolumn{2}{|l|}{ Whole brain } & \multicolumn{2}{|c|}{ Intra left hemispheric } & \multicolumn{2}{|c|}{ Intra right hemispheric } & \multicolumn{2}{|c|}{ Inter hemispheric } \\
\hline & & M. \pm Std. & $p$-val. $(F)$ & M. \pm Std. & $p$-val. $(F)$ & M. \pm Std. & $p$-val. $(F)$ & M. \pm Std. & $p$-val. $(F)$ \\
\hline Alpha & $\begin{array}{l}\text { Normal } \\
\mathrm{MCl}\end{array}$ & $\begin{array}{l}1.121 \pm .041 \\
1.105 \pm .036\end{array}$ & $.0068^{* *}(8.3)$ & $\begin{array}{l}1.091 \pm .040 \\
1.074 \pm .033\end{array}$ & $.052(4.06)$ & $\begin{array}{l}1.037 \pm .045 \\
1.029 \pm .031\end{array}$ & $.1664(2.0)$ & $\begin{array}{l}1.078 \pm .045 \\
1.066 \pm .033\end{array}$ & $.1069(2.74)$ \\
\hline Theta & $\begin{array}{l}\text { Normal } \\
\mathrm{MCI}\end{array}$ & $\begin{array}{l}.867 \pm .050 \\
.832 \pm .039\end{array}$ & $5.6533 \times 10^{-5^{* *}}(21.15)$ & $\begin{array}{l}.710 \pm .041 \\
.693 \pm .029\end{array}$ & $7.4952 \times 10^{-5^{* *}}(20.3)$ & $\begin{array}{l}1.023 \pm .053 \\
.971 \pm .037\end{array}$ & $.5366(.39)$ & $\begin{array}{l}.994 \pm .057 \\
.933 \pm .052\end{array}$ & $.0026^{* *}(10.6)$ \\
\hline
\end{tabular}

* Correlation is significant at the 0.05 level.

** Correlation is significant at the 0.01 level.

of intra left hemispheric network at theta and (d) $C_{r}$ of inter hemispheric network at theta. In all cases $C_{r}$ is decreased in $\mathrm{MCI}$ subjects.

\subsection{Efficiency Complexity $C_{e}$}

The same results with slightly better $p$-values were obtained by $C_{e}$. Table 3 shows the mean and standard deviation of $C_{e}$ of the whole brain, intra left and right hemispheric, and inter hemispheric neuronal networks at alpha and theta bands, as well as the associated ANOVA $p$-values and $F$-values in discrimination of the $\mathrm{MCI}$ and healthy elderly groups. It is observed that at alpha band only $C_{e}$ of the hole brain is significantly different between the two groups ( $p$-value $<0.01$ ), while at theta band the ability of $C_{e}$ in the whole brain and intra left hemisphere in distinguishing the two groups is much higher ( $p$-value $<0.0001$ ). Also at the theta band $C_{e}$ of the inter-hemispheric network is significantly decreased in $\mathrm{MCI}$ group ( $p$-value $<0.01)$. Fig. 6 shows distribution of $C_{e}$ of the brain neuronal network at (a) alpha and (b) theta; (c) $C_{e}$ of intra left hemispheric network at theta and (d) $C_{e}$ of inter hemispheric network at theta. In all cases the $C_{e}$ is decreased in $\mathrm{MCI}$ subjects. 
a

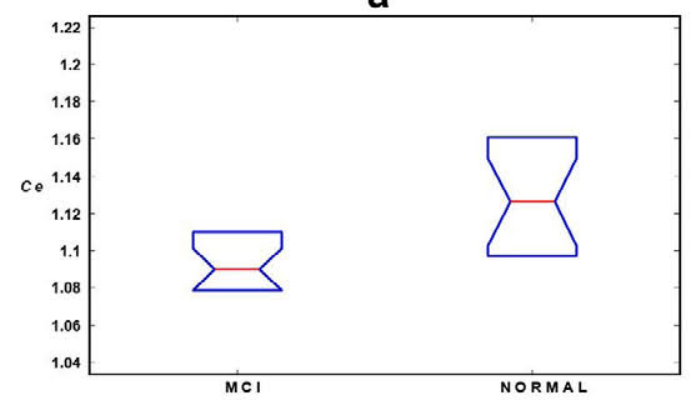

C

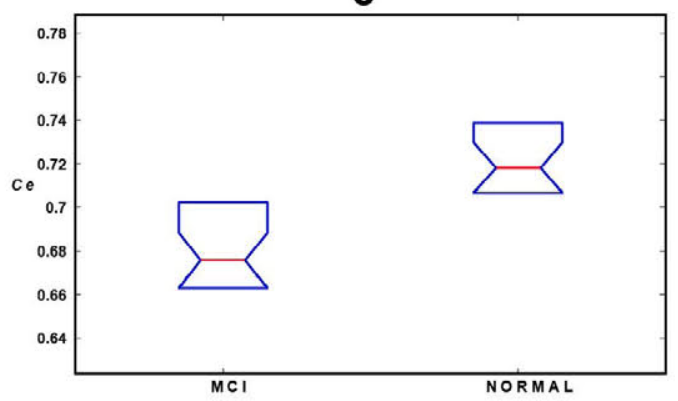

b

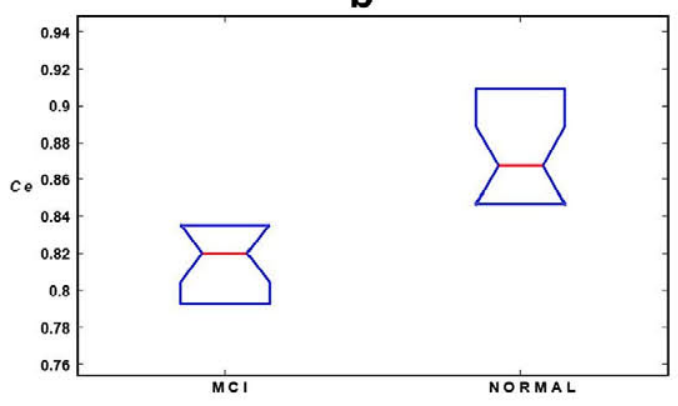

d

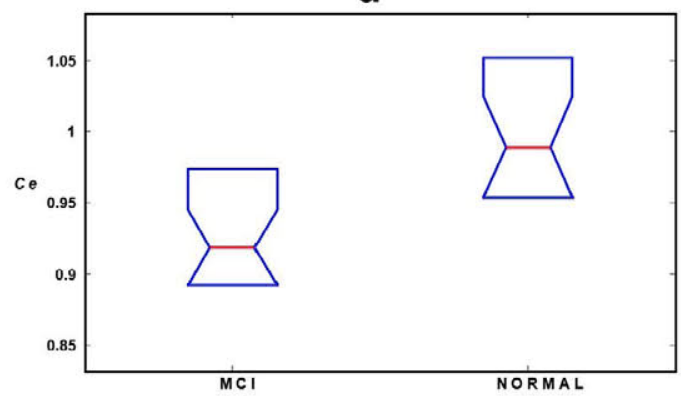

Fig. 6. $C_{e}$ of brain neuronal network at (a) alpha and (b) theta; (c) $C_{e}$ of intra left hemispheric network at theta and (d) $C_{e}$ of inter hemispheric network at theta.

The complexity of the brain network $\left(C_{e}\right.$ and $\left.C_{r}\right)$ was computed in other subbands, delta $(0.5-4 \mathrm{~Hz})$, beta $(13-30 \mathrm{~Hz})$, and gamma $(30-70 \mathrm{~Hz}))$ as well. The results did not indicate any meaningful difference.

\subsection{Classification}

Since the ANOVA tests showed $C_{e}$ of the whole brain and intra left hemisphere at theta band are the most discriminating features (see Tables 2 and 3), the classification was accomplished based on those two features. There are three standard methods of cross-validation: Leave-one-out, K-fold, and repeated random sub-sampling. We used the repeated random sub-sampling cross validation method, because based on our previous studies we found it more reliable than others.

A cross validation of repeated random sub-sampling of data with testing-to-training data ratio of 20-80 was performed over 100 repetitions to report reliably accurate classification results. An average accuracy of $97.6 \%$ was obtained using the EPNN classifier with sensitivity of $99.2 \%$ and specificity of $94.8 \%$.

It should be noted that a conventional method of classification, that is, the linear discriminant analysis (LDA) was also employed in this research. An accuracy of $92.8 \%$ was obtained, substantially less than that obtained by the EPNN.

\section{Discussion and conclusion}

Impaired working memory is a major problem in $\mathrm{MCl}$, especially in amnestic MCI. Previous studies have investigated various local brain networks and regions associated with impaired working memory in subjects with MCI. Decreasing posterior alpha power in MCI patients (Babiloni et al., 2008), gray matter loss in temporal, parietal, and frontal regions (Karas et al., 2004; Barbeau et al., 2008) and increased activity of occipital regions in MCI subjects during incremental learning (Moulin et al., 2007) are some of the major findings previously reported. Although there are some studies on functional connectivity of MCl's brain (Gómez et al., 2009;
Escudero et al., 2011), only a few researchers have investigated the global organization of brain networks in $\mathrm{MCl}$ (de Haan et al., 2012).

Several studies indicate that an increase in the task difficulty requires further synchronization of the brain network (Korobeinikova and Karatygin, 2012; Bernarding et al., 2013). The authors hypothesize that $\mathrm{MCI}$ subjects are more inefficient in the task execution. They need to make a greater effort to perform the same task.

In this paper, in order to study global variations in the working memory of the $\mathrm{MCI}$ brain relative to the normal brain, the authors examined the complexity of functional networks of whole, interhemispheric, and intra-hemispheric brain of $\mathrm{MCI}$ subjects during the Sternberg task and compared it with those of normal subjects. The networks' complexities were measured by Graph Index Complexity $\left(C_{r}\right)$ and Efficiency Complexity $\left(C_{e}\right)$. As theta and alpha bands are well-known frequencies involved in working memory functions, the $C_{r}$ and $C_{e}$ were computed and investigated in these frequency bands for both $\mathrm{MCI}$ and normal subjects.

The results obtained by both measurements $\left(C_{r}\right.$ and $\left.C_{e}\right)$ showed complexity of functional networks involved in the working memory function in $\mathrm{MCI}$ subjects were reduced at both alpha and theta bands compared with subjects with normal cognition and at the theta band this reduction is more pronounced in the whole brain and intra left hemisphere. This means the connectivity of the functional brain network in MCI subjects is such that the network has less efficiency with slower information transmission during the verbal working memory task. Since the verbal and phonological information is processed mainly in the left hemisphere, a possible explanation of finding more deficits in the left hemisphere in our study is that we used a verbal task. Also, the results show that $C_{e}$ would be a better measurement for showing the global differences between normal and $\mathrm{MCI}$ brains (with $p$-values less than 0.0001) compared with $C_{r}$. Furthermore, the high accuracy of the classification shows $C_{e}$ at theta band can be used as an index for assessing deficits associated with working memory, a good biomarker for diagnosis of MCI. Further studies are needed to determine if this index can be used in the pre-MCI stage to determine which individ- 
uals with normal cognition will go onto develop $\mathrm{MCl}$ as this would be useful for prognostication and for the institution of therapies once they become available. The results show the differences of the network complexity between the two groups are more significant in the left intra-hemispheric network relative to the right one. This may be explained by the fact that semantic processing and also word learning are more associated with left hemisphere (especially left prefrontal cortex and left temporal cortex) and hence it is why complexity of the left intra-hemispheric network during the Sternberg task has been changed much more in $\mathrm{MCI}$, compared with the right intra-hemispheric network (Gronholm et al., 2007; Clement et al., 2010).

Since the Efficiency Complexity in the $\mathrm{MCI}$ group is clearly lower than in the Control Group this research verifies the authors' hypothesis. Both groups are performing comparably in the Sternberg task but with a different efficiency.

As noted in the Section 1, Ahmadlou and Adeli (2011a) presented a new methodology for investigation of the structure of the brain functional connectivity based on graph theory and community pattern analysis of functional connectivity graph of the brain obtained from EEGs. From the viewpoint of Functional Connectivity analysis, compared with EEGs where one specific channel is needed as the reference for the other channels the main advantage of MEG records is no reference channel is needed (see Knake, 2006; Leahy et al., 1998). Other advantages are that MEG presents a better signal to noise ratio especially in the high frequency bands, and the fact that biological tissue is much more transparent (see Muthukumaraswamy, 2013 for a review) to magnetic fields (MEG) than to EEGs, thus making MEG a bit more sensitive. Finally, the authors' overarching research ideology advances a multi-modal measurement and analysis approach as the most effective way to investigate the complex brain system."

Only one set of five letters was used. This would substantially affect the interpretation. Consequently, one may consider the task applied to be an atypical short-term memory task. Considering the relatively small size of the data sample $(18+19$ subjects $)$, the results may be considered preliminary. Additional studies are needed to validate the consistency of the outcomes presented in this article.

\section{References}

Adeli H, Ghosh-Dastidar S, Dadmehr N. Alzheimer's disease and models of computation: imaging, classification, and neural models. J Alzheimers Dis 2005a; 7:187-99.

Adeli H, Ghosh-Dastidar S, Dadmehr N. Alzheimer's disease: models of computation and analysis of EEGs. Clin EEG Neurosci 2005b;36:131-40.

Adeli H, Ghosh-Dastidar S, Dadmehr N. A spatio-temporal wavelet-chaos methodology for EEG-based diagnosis of Alzheimer's disease. Neurosci Lett 2008:444:190-4

Adeli H, Panakkat A. A probabilistic neural network for earthquake magnitude prediction. Neural Netw 2009;22:1018-24.

Ahmadlou M, Adeli $\mathrm{H}$. Enhanced probabilistic neural network with local decision circles: a robust classifier. Integr Comput-Aid E 2010a;17:197-210.

Ahmadlou M, Adeli H. Wavelet-synchronization methodology: a new approach for EEG-based diagnosis of ADHD. Clin EEG Neurosci 2010b;41:1-10.

Ahmadlou M, Adeli H. Functional community analysis of brain: a new approach for EEG-based investigation of the brain pathology. Neurolmage 2011a;58:401-8.

Ahmadlou M, Adeli H. Fuzzy synchronization likelihood with application to attention-deficit/hyperactivity disorder. Clin EEG Neurosci 2011b;42:6-13.

Ahmadlou M, Adeli $\mathrm{H}$. Visibility graph similarity: a new measure of generalized synchronization in coupled dynamic systems. Physica D 2012;241:326-32.

Ahmadlou M, Adeli H. Adeli A. New diagnostic EEG markers of the Alzheimer's disease using visibility graph. J Neural Transm 2010;117:1099-109.

Ahmadlou M, Adeli H, Amir A. Graph theoretical analysis of organization of functional brain networks in ADHD. Clin EEG Neurosci 2012a;43:5-13.

Ahmadlou M, Adeli H, Adeli A. Improved visibility graph fractality with application for diagnosis of autism spectrum disorder. Physica A 2012b;391:4720-6.

Ahmadlou M, Adeli H, Adeli A. Fractality analysis of frontal brain in major depressive disorder. Int J Psychophysiol 2012c;85:206-11.

Ahmadlou M, Adeli H, Adeli A. Fuzzy synchronization likelihood-wavelet methodology for diagnosis of autism spectrum disorder. J Neurosci Methods 2012d;211:203-9.
Ahmadlou M, Ahmadi K, Rezazade M, Azad-Marzabadi E. Global organization of functional brain connectivity in methamphetamine abusers. Clin Neurophysiol 2013;124:1122-31.

Ally BA, McKeever JD, Waring JD, Budson AE. Preserved frontal memorial processing for pictures in patients with mild cognitive impairment. Neuropsychologia 2009; 47:2044-55.

Babiloni C, Frisoni GB, Pievani M, Toscano L, Del Percio C, Geroldi C, et al. Whitematter vascular lesions correlate with alpha EEG sources in mild cognitive impairment. Neuropsychologia 2008;46:1707-20.

Baddeley AD, Hitch G. Working memory. In: Bower GA, editor. The psychology of learning and motivation: advances in research and theory 1974;vol. 8. New York: Academic Press; 1974. p. 47-89.

Bajo R, Maest F, Nevado A, Sancho M, Gutiérrez R, Campo P, et al. Functional connectivity in mild cognitive impairment during a memory task: implications for the disconnection hypothesis. J Alzheimers Dis 2010;22: $183-93$.

Bajo R, Castellanos NP, Cuesta P, Aurtenetxe S, Garcia-Prieto J, Gil-Gregorio P, et al. Differential patterns of connectivity in progressive mild cognitive impairment. Brain Connect 2012;2:21-4.

Barbeau EJ, Ranjeva JP, Didic M, Confort-Gouny S, Felician O, Soulier E, et al. Profile of memory impairment and gray matter loss in amnestic mild cognitive impairment. Neuropsychologia 2008;46:1009-19.

Barrat A, Barthelemy M, Pastor-Satorras R, Vespignani A. The architecture of complex weighted networks. PNAS 2004;101:3747-52.

Bastiaansen MCM, Posthuma D, Groot PFC, de Geus EJC. Event-related alpha and theta responses in a visuo-spatial working memory task. Clin Neurophysiol 2002;113:1882-93.

Bello-Orgaz G, Menendez H, Camacho D. Adaptive K-means algorithm for overlapped graph clustering. Int J Neural Syst 2012;22:1250018.

Bernarding C, Strauss DJ, Hannemann R, Seidler H, Corona-Strauss FI. Neural correlates of listening effort related factors: influence of age and hearing impairment. Brain Res Bull 2013;91:21-30.

Besio WG, Liu X, Wang L, Medvedev AV, Koka K. Transcutaneous focal electrical stimulation via concentric ring electrodes reduces synchrony induced by pentylenetetrazole in beta and gamma bands in rats. Int J Neural Syst 2011;21:139-49.

Brookes MJ, Wood JR, Stevenson CM, Zumer JM, White TP, Liddle PF, et al. Changes in brain network activity during working memory tasks: a magnetoencephalography study. Neuroimage 2011;55:1804-15.

Choi EH, Jung KI, Jun AY, Kang SW. Clinical characteristics of patients in a dementia prevention center. J Neurol Sci 2009;283:291-2.

Clement F, Belleville S, Mellah S. Functional neuroanatomy of the encoding and retrieval processes of verbal episodic memory in $\mathrm{MCl}$ Cortex 2010;46:1005-15.

Collie A, Maruff P. The neuropsychology of preclinical Alzheimer's disease and mild cognitive impairment. Neurosci Biobehav Rev 2000;24:365-74.

Deeny SP, Poeppel D, Zimmerman JB, Roth SM, Brandauer J, Witkowski S, et al. Exercise, APOE, and working memory: MEG and behavioral evidence for benefit of exercise in epsilon4 carriers. Biol Psychol 2008;78:179-87.

de Haan W, Mott K, van Straaten EC, Scheltens P, Stam CI. Activity dependent degeneration explains hub vulnerability in Alzheimer's disease. PLoS Comput Biol 2012;8:e1002582.

Deiber MP, Ibanez V, Missonnier P, Herrmann F, Fazio-Costa L, Gold G, et al. Abnormal-induced theta activity supports early directed-attention network deficits in progressive MCI. Neurobiol Aging 2009;30:1444-52.

deToledo-Morrell L, Evers S, Hoeppner TJ, Morrell F, Garron DC, Fox JH. A ‘stress' test for memory dysfunction: electrophysiologic manifestations of early Alzheimer's disease. Arch Neurol 1991;48:605-9.

Escudero J, Sanei S, Jarchi D, Absolo D, Hornero R. Regional coherence evaluation in mild cognitive impairment and Alzheimer's disease based on adaptively extracted magnetoencephalogram rhythms. Physiol Meas 2011;32:1163-80.

Gómez C, Stam CJ, Hornero R, Fernndez A, Maest F. Disturbed beta band functional connectivity in patients with mild cognitive impairment: an MEG study. IEEE Trans Biomed Eng 2009;56:1683-90.

Gronholm P, Rinne JO, Vorobyev VA, Laine M. Neural correlates of naming newly learned objects in MCI. Neuropsychologia 2007;45:2355-68.

Grundman M, Petersen RC, Ferris SH, Thomas RG, Aisen PS, Bennett DA, et al. Mild cognitive impairment can be distinguished from Alzheimer disease and normal aging for clinical trials. Arch Neurol 2004;61:59-66.

Han F, Wiercigroch M, Fang JA, Wang Z. Excitement and synchronization of smallworld neuronal networks with short-term synaptic plasticity. Int J Neural Syst 2011;21:415-25.

Karas G, Scheltens P, Rombouts S, Visser P, van Schijndel R, Fox N, et al. Global and local gray matter loss in mild cognitive impairment and Alzheimer's disease. Neuroimage 2004;23:708-16.

Khader PH, Jost K, Ranganath C, Rosler F. Theta and alpha oscillations during working-memory maintenance predict successful long-term memory encoding. Neurosci Lett 2010;468:339-43.

Kim J, Wilhelm T. What is a complex graph? Physica A 2008;387:2637-52.

Klimesch W. EEG alpha and theta oscillations reflect cognitive and memory performance: a review and analysis. Brain Res Rev 1999;29:169-95.

Knake S, Halgren E, Shiraishi H, Hara K, Hamer HM, Grant PE, et al. The value of multichannel MEG and EEG in the presurgical evaluation of 70 epilepsy patients. Epilepsy Res 2006;69:80-6. 
Korobeinikova II, Karatygin NA. Spectral-coherent characteristics of EEG alpha rhythm in different efficiency of visual spatial task performance in humans. Bull Exp Biol Med 2012;153:601-4

Kurimoto R, Ishii R, Canuet L, Ikezawa K, Iwase M, Azechi M, et al. Induced oscillatory responses during the Sternberg's visual memory task in patients with Alzheimer's disease and mild cognitive impairment. Neuroimage 2012;59:4132-40.

Latora V, Marchiori M. Economic small-world behavior in weighted networks. Eur Phys J B 2003;32:249-63.

Leahy RM, Mosher JC, Spencer ME, Huang MX, Lewine JD. A study of dipole localization accuracy for MEG and EEG using a human skull phantom. Electroencephalogr Clin Neurophysiol 1998;107:159-73.

Li W, Lin Y, Liu Y. The structure of weighted small-world networks. Physica A 2007;376:708-18.

Lobo A, Ezquerra J, Gomez BF, Sala JM, Seva DA. Cognocitive mini-test (a simple practical test to detect intellectual changes in medical patients). Actas Luso Esp Neurol Psiquiatr Cienc Afines 1979;7:189-202.

Lopez-Rubio E, Luque-Baena RM, Dominguez E. Foreground detection in video sequences with probabilistic self-organizing maps. Int J Neural Syst 2011;21:225-46.

Maest F, Fernandez A, Simos PG, Gil-Gregorio P, Amo C, Rodriguez R, et al. Spatiotemporal patterns of brain magnetic activity during a memory task in Alzheimer's disease. Neuroreport 2001;12:3917-22.

Maest F, Campo P, Del Ro D, Moratti S, Gil-Gregorio P, Fernndez A, et al. Increased biomagnetic activity in the ventral pathway in mild cognitive impairment. Clin Neurophysiol 2008;119:1320-7.

Mitchell AJ. Is it time to separate subjective cognitive complaints from the diagnosis of mild cognitive impairment? Age Ageing 2008;37:497-9.

Morgan HM, Jackson MC, van Koningsbruggen MG, Shapiro KL, Linden DEJ. Frontal and parietal theta burst TMS impairs working memory for visual-spatial conjunctions. Brain Stimul 2013;6:122-9.

Moulin CJ, Laine M, Rinne JO, Kaasinen V, Sipilä H, Hiltunen J, et al. Brain function during multi-trial learning in mild cognitive impairment: a PET activation study. Brain Res 2007;1136:132-41.

Muthukumaraswamy SD. High frequency brain activity and muscle artifacts in MEG/EEG: a review and recommendations. Front Hum Neurosci 2013. http:/ dx.doi.org/10.3389/fnhum.2013.00138.

Patalong-Ogiewa MB, Siuda JS, Opala GM. Working and episodic memory in the MCI group. J Neurol Sci 2009;283:291.

Petersen RC. Mild cognitive impairment as a diagnostic entity. J Intern Med $2004 ; 256: 183-94$.
Petersen RC, Doody R, Kurz A, Mohs RC, Morris JC, Rabins PV, et al. Current concepts in mild cognitive impairment. Arch Neurol 2001;58:1985-92.

Ramanathan K, Ning N, Dhanasekar D, Guoqi L, Luping S, Vadakkepat P. Presynaptic learning and memory with a persistent firing neuron and a habituating synapse: A model of short term persistent habituation. Int J Neural Syst 2012;22:1250015.

Rangaprakash D, Hu X, Deshpande G. Phase synchronization in brain networks derived from correlation between probabilities of Recurrences in functional MRI data. Int J Neural Syst 2013;23:1350003.

Sajedi F, Ahmadlou M, Vameghi R, Gharib M, Hemmati S. Linear and nonlinear analysis of brain dynamics in children with cerebral palsy. Res Dev Disabil 2013;34:1388-96.

Schmiedt C, Brand A, Hildebrandt H, Basar-Eroglu C. Event-related theta oscillations during working memory tasks in patients with schizophrenia and healthy controls. Cognitive Brain Res 2005;25:936-47.

Serletis D, Carlen PL, Valiante TA, Bardakjian BL. Phase synchronization of neuronal noise in mouse Hippocampal epileptiform dynamics. Int J Neural Syst 2013;23:1250033.

Stam CJ. Brain dynamics in theta and alpha frequency bands and working memory performance in humans. Neurosci lett 2000;286:115-8.

Stam CJ, van Dijk BW. Synchronization likelihood: an unbiased measure of generalized synchronization in multivariate data sets. Physica D 2002;163:236-51.

Stephane M, Ince NF, Kuskowski M, Leuthold A, Tewfik AH, Nelson K, et al. Neura oscillations associated with the primacy and recency effects of verbal working memory. Neurosci Lett 2010;473:172-7.

Sternberg S. High-speed scanning in human memory. Science 1966;153:652-4.

Tuladhar AM, ter Huurne N, Schoffelen JM, Maris E, Oostenveld R, Jensen O. Parietooccipital sources account for the increase in alpha activity with working memory load. Hum Brain Mapp 2007;28:785-92.

Villeneuve S, Belleville S, Lacombe J, Labelle MA, Enriquez-Rosas A, Gauthier S Episodic memory deficits in vascular and non vascular mild cognitive impairment. J Neurol Sci 2009;283:291.

Watts DJ, Strogatz SH. Collective dynamics of 'small-world' networks. Nature 1998;393:409-10.

Wenger MJ, Negash S, Petersen RC, Petersen L. Modeling and estimating recal processing capacity: sensitivity and diagnostic utility in application to mild cognitive impairment. J Math Psychol 2010;54:73-89.

Yesavage JA, Brooks JO. On the importance of longitudinal research in Alzheimer's disease. J Am Geriatr Soc 1991;39:942-9. 